\title{
Cumplimiento con la legislación de ambientes libres de humo de tabaco en México
}

\author{
Tonatiuh Barrientos-Gutiérrez, MD, M en C, ${ }^{(1,2)}$ Luz Myriam Reynales-Shigematsu, MD, M en C, D en C,(1) \\ David Gimeno, PhD, (3) Eduardo Lazcano-Ponce, MD, M en C, D en C. (I)
}

\author{
Barrientos-Gutiérrez T, Reynales-Shigematsu LM, \\ Gimeno D, Lazcano-Ponce E. \\ Cumplimiento con la legislación de ambientes \\ libres de humo de tabaco en México. \\ Salud Publica Mex 2008;50 supl 3:S31 5-S322.
}

\begin{abstract}
Resumen
Este texto pretende establecer criterios para identificar espacios libres de humo de tabaco ambiental (HTA) utilizando monitoreo, inspección directa y reporte de los trabajadores, comparando su capacidad de discriminación y derivando una propuesta con aplicación a un sistema de vigilancia epidemiológica. En 10 instituciones se monitoreó nicotina ambiental, realizando inspecciones y obteniendo el reporte de los trabajadores sobre la presencia de HTA. Para cada método se definieron criterios para clasificar a las instituciones como espacios libres de HTA. Los resultados fueron comparados para analizar el nivel de acuerdo entre métodos. Se observó buen acuerdo entre monitoreo ambiental e inspección directa, discrepando en $20 \%$ de las decisiones. El reporte de los trabajadores fue demasiado frecuente para discriminar. La combinación del monitoreo ambiental con la inspección proporcionaría la clasificación más sensible. Se requieren estudios de costo-efectividad para identificar la mejor estrategia.
\end{abstract}

Palabras clave: vigilancia epidemiológica; contaminación por humo de tabaco; cese del uso de tabaco; legislación; México
Barrientos-Gutiérrez T, Reynales-Shigematsu LM, Gimeno D, Lazcano-Ponce E.

Compliance with the tobacco smoke

free ambience legislation in Mexico.

Salud Publica Mex 2008;50 suppl 3:S3 I5-S322.

\begin{abstract}
This essay tries o develop classification criteria to identify smoke-free spaces using environmental monitoring, direct inspection and worker reports, comparing their agreement and deriving a proposal useful for the epidemiological surveillance of environmental tobacco smoke. Environmental nicotine monitoring, direct inspections and worker's surveys regarding tobacco smoke presence were conducted in ten institutions. For each method, criteria were defined to classify institutions as smoke-free spaces. Results were compared to evaluate between-methods agreement. Good agreement between environmental monitoring and direct inspections were observed, although they disagreed in $20 \%$ of the cases. Worker reports were too frequent to discriminate. Combining environmental monitoring and inspection would provide the most sensitive classification. Cost-effectiveness studies are required to identify the best strategy.
\end{abstract}

Key words: epidemiological surveillance; tobacco smoke pollution; tobacco use cessation; legislation; Mexico

(I) Centro de Investigación en Salud Poblacional, Instituto Nacional de Salud Pública, Cuernavaca, México.

(2) The University of Texas School of Public Health, Division of Environmental and Occupational Health Sciences, Health Science Center at Houston, Texas, USA.

(3) International Institute for Society and Health, Department of Epidemiology and Public Health, University College London, London, England, United Kingdom.

Fecha de aceptado: 14 de mayo de 2008

Solicitud de sobretiros: M en C Tonatiuh Barrientos Gutiérrez. Departamento de Investigación sobre Tabaco. Instituto Nacional de Salud Pública. $7^{\mathrm{a}}$ Cerrada de Fray Pedro de Gante Num. 50, col Sección XVI, C.P. I4000, del. Tlalpan, México DF.

Correo electrónico: tbarrientos@correo.insp.mx 
L a Organización Mundial de la Salud (OMS) desarrolló en 2003 el Convenio Marco para el Control del Tabaco (CMCT), ${ }^{1}$ el cual fue ratificado por México un año después. ${ }^{2}$ El CMCT constituye el primer instrumento jurídico internacional para reducir globalmente la morbimortalidad atribuible al tabaquismo activo y pasivo. El CMCT establece principios para regular los productos del tabaco a través del control de la publicidad, promoción, patrocinio, empaquetado, tasación y tráfico ilícito. Además, establece los lineamientos para la protección de los no fumadores hacia la exposición al humo de tabaco ambiental (HTA). El HTA contiene más de 250 substancias tóxicas y carcinogénicas y es un riesgo comprobado para la salud. ${ }^{3}$ Dado su potencial nocivo, no es posible establecer valores límite de exposición, siendo riesgoso para la salud en cualquier cantidad. ${ }^{4}$ En consecuencia, la OMS propuso la implementación de espacios $100 \%$ libres de humo, considerándolos como la única solución efectiva para eliminar la exposición al HTA en espacios interiores. ${ }^{5}$

En respuesta a los compromisos adquiridos tras la ratificación del CMCT en febrero de 2008, se aprobó la Ley General para el Control del Tabaco (LGCT), que establece las bases para la implementación de espacios libres de humo de tabaco en México. ${ }^{6}$ Según el Artículo 26 de la LGCT: "queda prohibido el consumo de cualquier producto del tabaco en todo lugar cerrado de acceso al público, salvo en los lugares permitidos específicamente para ello por los reglamentos aplicables", puntualizando que los espacios permitidos deberán ubicarse al aire libre, no ser áreas de paso obligado para usuarios y contar con mecanismos que eviten la contaminación de los espacios interiores.

Una vez desarrollado el marco legal, el reto inmediato es la implementación de la legislación, incluyendo la evaluación y vigilancia de su cumplimiento. En este contexto, el desarrollo de un sistema de vigilancia epidemiológica del HTA es fundamental para asegurar el éxito de los espacios libres de humo de tabaco. El sistema debe permitir la generación continua de información, facilitando la detección temprana de exposición, la evaluación de las políticas públicas a corto y mediano plazo y la identificación de espacios de oportunidad para mejorar el cumplimiento. El sistema de vigilancia debe estar basado en criterios bien fundamentados, de manera que se asegure la estabilidad de los métodos de evaluación a través del tiempo y de los diversos contextos geográficos de México, posibilitando comparaciones entre lugares y momentos.

En el ámbito internacional, la vigilancia epidemiológica de los espacios libres de HTA ha utilizado tres fuentes básicas de información: monitoreo ambiental, 7,8 inspecciones directas ${ }^{9-11} \mathrm{y}$ encuestas a usuarios de los espacios. ${ }^{12-14}$ La nicotina es el marcador preferido para monitoreo de HTA dada su alta especificidad (sólo es producida con la combustión del tabaco) y relativo bajo costo. ${ }^{15,16}$ La inspección directa se ha basado en tres indicadores: presencia de fumadores, detección de olor a humo de tabaco y presencia de colillas o ceniceros en el área evaluada. Finalmente, las encuestas a usuarios se han utilizado de manera masiva en la evaluación del cumplimiento con la legislación, particularmente para comparar la prevalencia de exposición reportada a HTA antes y después de la implementación de las restricciones.

De acuerdo con estos indicadores, en espacios libres de HTA: a) el monitoreo ambiental no debería detectar nicotina, b) no debería observarse a personas fumando, encontrarse colillas de cigarros o detectarse olor a taba$\mathrm{co}, \mathrm{y}$ c) ningún usuario debería reportar HTA en su área de trabajo. Sin embargo, existe incertidumbre acerca del grado de certeza que cada uno de estos métodos tiene para identificar el cumplimiento. En nuestra búsqueda en la literatura no encontramos artículos que simultáneamente utilizaran estos tres métodos y compararan su eficacia para la evaluación de cumplimiento. Por lo tanto, es necesario analizar estos tres métodos de forma separada y concurrente para identificar criterios sensibles para la evaluación de cumplimiento.

El objetivo de este estudio es proponer criterios para la clasificación del cumplimiento con los espacios 100\% libres de humo de tabaco, utilizando de forma independiente monitoreo ambiental, inspección directa y reporte de los trabajadores, comparando su capacidad de clasificación y derivando una propuesta integrada de evaluación para el desarrollo del sistema de vigilancia de la exposición al HTA en México.

\section{Material y métodos}

Durante 2006 fueron invitadas a participar 15 instituciones en las ciudades de Monterrey, Guadalajara y el Distrito Federal, que en su mayoría se encontraban localizadas en uno o más edificios de una o más plantas. Las instituciones invitadas pertenecían a tres tipos de espacio público: establecimientos relacionados con la salud (clínicas y hospitales), la educación (instituciones educativas de nivel medio y superior) y la administración (oficinas del gobierno). Diez de las instituciones contactadas (66.6\% respuesta) accedieron a participar, de las cuales siete eran consideradas libres de humo por sus representantes legales. Se solicitó permiso escrito a los representantes legales de las instituciones para acceder a las instalaciones y llevar a cabo los procedimientos de 
estudio, obteniendo los planos de construcción para las áreas donde se realizarían la inspección y el monitoreo ambiental, y la lista de trabajadores de las instituciones para seleccionar a los participantes en la encuesta. En los casos (40\%) en los que los planos de construcción no estuvieron disponibles, se generaron diagramas generales para identificar las áreas.

\section{Monitoreo}

El monitoreo fue efectuado utilizando monitores pasivos de nicotina en fase de vapor, cartuchos de plástico que contienen un filtro impregnado en bisulfato de sodio con una membrana que permite el paso del aire a una tasa constante $(24 \mathrm{ml} / \mathrm{min}) \cdot{ }^{17}$ Los niveles de nicotina se cuantificaron mediante cromatografía de gases con detección selectiva de nitrógeno realizada en el Laboratorio Analítico de Compuestos del Tabaco (LACOT) del Instituto Nacional de Salud Pública (INSP). El nivel de detección de nicotina fue establecido en $0.009 \mu \mathrm{g} / \mathrm{m}^{3}$.

En cada edificio disponible para el monitoreo se realizó un censo de áreas cerradas, definidas como áreas sin conexión con espacios adyacentes una vez cerradas puertas y ventanas. Tras levantar el censo se procedió a realizar un muestreo sistemático de $20 \%$ de las áreas para efectuar el monitoreo. El tiempo de monitoreo fue obtenido a partir de un piloto en dos cafeterías y un almacén de abastecimiento donde se había observado la presencia de consumo activo de tabaco. En cada área se colocaron tres grupos de tres monitores de nicotina que se activaron simultáneamente, exponiéndolos por ocho horas, siete o 14 días. Todos los monitores de siete y 14 días registraron niveles por encima del nivel de detección, pero sólo un grupo de los expuestos durante ocho horas registró tales niveles. Dado el menor costo y mayor facilidad operativa se prefirió el monitoreo de siete días para el presente estudio. Así, en cada área seleccionada se colocó un monitor, registrando día y hora de colocación y manteniéndolo continuamente expuesto por siete días. En cada espacio público se colocaron $10 \%$ de monitores duplicados y blancos para efectos de control de calidad.

Utilizando el monitoreo ambiental un espacio sólo podría clasificarse como libre de humo si ningún monitor sobrepasa los niveles de detección de nicotina. Sin embargo, en la práctica existe la posibilidad de contaminación de áreas interiores desde fuentes externas. Por ejemplo, el caso de fumadores a la entrada de los edificios, la exhalación de humo de tabaco en ambiente interior al entrar en un edificio o la contaminación a través de ventanas abiertas al exterior. En consecuencia, desarrollamos un criterio de cumplimiento considerando la posibilidad de contaminación exterior, estableciendo que en un espacio libre de humo de tabaco:

1. Las concentraciones de nicotina deben ser menores de $0.1 \mu \mathrm{g} / \mathrm{m}^{3}$ en al menos $90 \%$ de las áreas monitoreadas. Este punto de corte corresponde a la concentración mínima observada en áreas de no fumar de restaurantes sin separación física de las áreas de fumar. ${ }^{18}$ Aunque la decisión natural de punto de corte debería situarse en el límite de detección (detectable vs. no detectable) utilizar $0.1 \mu \mathrm{g} / \mathrm{m}^{3}$ incrementa la certeza de que las concentraciones que están siendo detectadas realmente representan contaminación derivada del consumo de tabaco en interiores y no artefactos de contaminación externa.

2. En ningún caso puede haber concentraciones mayores a $0.5 \mu \mathrm{g} / \mathrm{m}^{3}$. Ésta es la concentración máxima tolerable en un área de no fumar que pudiera estar contaminada por una fuente externa. Derivamos este punto de corte con base en un estudio previo, donde las concentraciones máximas detectadas en áreas de hospitales y escuelas con prohibición de fumar fueron de 0.6 y $0.5 \mu \mathrm{g} / \mathrm{m}^{3}$, respectivamente. ${ }^{18}$

3. Los monitores que detectan niveles de nicotina entre 0.1 y $0.5 \mu \mathrm{g} / \mathrm{m}^{3}$ deben estar en un área susceptible de contaminación externa de acuerdo con los planos utilizados para la colocación. Consideramos que un monitor es susceptible de contaminación externa si: a) se encuentra en el piso de entrada y salida al exterior, b) se encuentra cerca de puertas o ventanas que dan al exterior, y c) en el conjunto de monitores cercanos no es posible identificar un patrón claro de contaminación por consumo activo de tabaco.

\section{Inspección directa}

En cada área monitoreada un técnico entrenado llevó a cabo inspecciones aplicando una lista de observación. La lista contenía información para identificar fuentes de ventilación, ventanas y puertas, presencia de personas fumando en el área, olor a humo de tabaco, ceniceros o colillas en el área y objetos con publicidad sobre el tabaco. El técnico visitó estas áreas en cinco ocasiones durante una semana: en el momento de la colocación y retiro de los monitores y durante tres visitas intermedias realizadas al azar. Para clasificar un área como libre de HTA durante la inspección no debía detectarse cualquiera de los siguientes eventos:

1. Presencia de personas fumando, $y / 0$

2. Olor a humo de tabaco y ceniceros/colillas en el área 


\section{Encuesta a los trabajadores}

A través de un muestreo aleatorio simple y teniendo en cuenta la prevalencia de tabaquismo en hombres $(40 \%)$ y mujeres (19\%) mexicanas se estimó el tamaño de muestra para cada una de las instituciones. La encuesta se aplicó a los trabajadores e incluyó información demográfica (sexo, edad, ocupación, puesto de trabajo), consumo de tabaco, presencia de HTA en el lugar de trabajo y percepción de políticas de control del tabaco. Para efectos de desarrollar un criterio de cumplimiento se utilizó la pregunta " ¿Cree usted que la cantidad de humo de tabaco en su área de trabajo es...?" con potenciales respuestas "muy alto", "alto", "medio", "bajo" y "nulo". Un área se clasificaría como libre de HTA si:

1. $\mathrm{Al}$ menos $75 \%$ de los trabajadores reportaba un nivel nulo de HTA

2. Menos de $10 \%$ de los trabajadores reportaba un nivel alto o muy alto de HTA

\section{Resultados}

El cuadro I describe las características principales de los espacios públicos participantes. La mitad de las instituciones contaba con un solo edificio y el resto entre 5 y 12 edificios. El número de áreas monitoreadas por edificio varió entre 7 y 69 según el tamaño de la institución. Excepto las universidades, las instituciones se consideraban espacios libres de humo de tabaco según el representante legal. Se encuestó un promedio de 170 trabajadores por institución y la prevalencia media de tabaquismo activo fue de 30 por ciento.

El cuadro II presenta los resultados del monitoreo. En todas las instituciones excepto el instituto de investigación se detectó nicotina, fluctuando desde 9 hasta $80 \%$ de las áreas en un hospital y la clínica de atención neumológica, respectivamente. En siete instituciones se detectaron concentraciones superiores a $0.1 \mu \mathrm{g} / \mathrm{m}^{3}$, aunque sólo en dos el porcentaje de monitores con estas concentraciones superó el 10 por ciento. En cuatro instituciones se detectaron concentraciones superiores a $0.5 \mu \mathrm{g} / \mathrm{m}^{3}$, siendo la concentración máxima $3.7 \mu \mathrm{g} / \mathrm{m}^{3}$, observada en un hospital. La evaluación de las áreas con concentraciones superiores a $0.1 \mu \mathrm{g} / \mathrm{m}^{3}$ pero menores a $0.5 \mu \mathrm{g} / \mathrm{m}^{3}$ utilizando el plano arquitectónico descartó únicamente el caso de la escuela preparatoria, donde se consideró que existía la posibilidad de contaminación por fuentes externas.

El cuadro III presenta los resultados de la inspección directa. Durante las inspecciones se observó a personas fumando en las áreas interiores de tres instituciones, olor a tabaco en seis instituciones y colillas en cuatro

\section{Cuadro I}

Descripción general de espacios públicos, PARA MONITOREO DE NICOTINA. MÉXICO, 2007

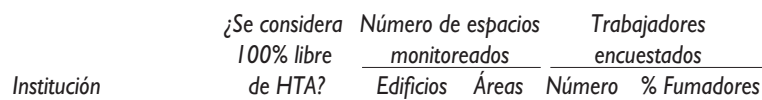

\begin{tabular}{lccrrr} 
Hospital I & Sí & I & 56 & 146 & 35 \\
\hline Hospital 2 & Sí & I & 69 & 95 & 20 \\
\hline Clínica de neumología & Sí & I & 10 & 41 & 17 \\
\hline Universidad I & No & 10 & 67 & 270 & 32 \\
\hline Universidad 2 & Sí & 12 & 55 & 246 & 29 \\
\hline Universidad 3 & No & 11 & 67 & 267 & 29 \\
\hline Universidad 4 & No & 6 & 23 & 254 & 5 I \\
\hline Preparatoria & Sí & 5 & 14 & 232 & 32 \\
\hline Instituto de investigación & Sí & I & 7 & 23 & 26 \\
\hline Oficinas & Sí & I & 69 & 120 & 33
\end{tabular}

HTA= humo de tabaco ambiental

Cuadro II

Resultados del MONITOREO AMBIENTAL DE NICOTINA EN FASE DE VAPOR eN ESPACIOS PÚBLICOS. MÉXICO, 2007

$\begin{array}{ccccc} & & \text { Nivel } & \text { ¿Posible } \\ \text { Institución } & \text { Porcentaje } & \text { Porcentaje } & \text { máximo } & \text { contaminación } \\ & >\text { ND* } & \geq 0.1 \mu \mathrm{g} / \mathrm{m} 3 & \text { observado } & \text { externa? }\end{array}$

\begin{tabular}{lrrll} 
Hospital I & 8.9 & 5.4 & 1.85 & No \\
\hline Hospital 2 & 15.9 & 7.2 & 3.7 & No \\
\hline Clínica de neumología & 80.0 & 0.0 & 0.049 & NA \\
\hline Universidad I & 17.9 & 0.0 & 0.016 & NA \\
\hline Universidad 2 & 52.7 & 3.6 & 0.1 & No \\
\hline Universidad 3 & 19.4 & 4.5 & 0.17 & No \\
\hline Universidad 4 & 47.8 & 21.7 & 1.04 & No \\
\hline Preparatoria & 28.6 & 7.1 & 0.32 & Si \\
\hline Instituto de investigación & 0.0 & 0.0 & $<\mathrm{ND}$ & $\mathrm{NA}$ \\
\hline Oficinas & 42.0 & 26.1 & 0.64 & $\mathrm{No}$
\end{tabular}

*ND: Nivel de detección $\left(0.009 \mu \mathrm{g} / \mathrm{m}^{3}\right)$

instituciones. La detección de personas fumando fue el indicador menos estable en el tiempo, ya que fue detectado en 20 a $60 \%$ de las visitas realizadas, mientras que olor a tabaco y colillas se observaron en 20 a 100\% de las visitas.

El cuadro IV presenta los resultados de la encuesta. Excepto en el caso del instituto de investigación, la 


\section{Cuadro III}

Resultados de LA INSPECCIÓN DIRECTA EN ESPACIOS PÚBlicos. MÉXICO, 2007

\begin{tabular}{|c|c|c|c|c|}
\hline \multirow[b]{2}{*}{ Institución } & \multicolumn{3}{|c|}{$\begin{array}{c}\text { Número (\%) de días en los que } \\
\text { se observa el evento }\end{array}$} & \multirow{2}{*}{$\begin{array}{c}\text { Total de } \\
\text { eventos } \\
\text { observados }\end{array}$} \\
\hline & $\begin{array}{l}\text { Personas } \\
\text { fumando }\end{array}$ & $\begin{array}{l}\text { Olor a humo } \\
\text { de tabaco }\end{array}$ & Colillas & \\
\hline Hospital I & $0(0)$ & $0(0)$ & $0(0)$ & 0 \\
\hline Hospital 2 & $0(0)$ & $5(100)$ & $5(100)$ & 2 \\
\hline Clínica de neumología & $0(0)$ & $0(0)$ & $0(0)$ & 0 \\
\hline Universidad I & $0(0)$ & $2(40)$ & $0(0)$ & I \\
\hline Universidad 2 & $2(40)$ & $3(60)$ & $4(80)$ & 3 \\
\hline Universidad 3 & $3(60)$ & $4(80)$ & $4(80)$ & 3 \\
\hline Universidad 4 & $0(0)$ & I (20) & $0(0)$ & 1 \\
\hline Preparatoria & $0(0)$ & $0(0)$ & $0(0)$ & 0 \\
\hline Instituto de investigación & $0(0)$ & $0(0)$ & $0(0)$ & 0 \\
\hline Oficinas & I (20) & $5(100)$ & $5(100)$ & 3 \\
\hline
\end{tabular}

\section{Cuadro IV}

Resultados de la encuesta de trabajadores EN ESPACIOS PÚBlicos. MÉXICO, 2007

\begin{tabular}{lcc} 
Institución & $\begin{array}{c}\text { Porcentaje de trabajadores } \\
\text { que reportan HTA } \\
\text { en su área de trabajo }\end{array}$ & $\begin{array}{c}\text { Porcentaje de trabajadores } \\
\text { que reportan alto HTA } \\
\text { en su área de trabajo }\end{array}$ \\
Hospital I & 68 & 43 \\
\hline Hospital 2 & 46 & 22 \\
\hline Clínica de neumología & 34 & 20 \\
\hline Universidad I & 71 & 45 \\
\hline Universidad 2 & 83 & 59 \\
\hline Universidad 3 & 66 & 42 \\
\hline Universidad 4 & 72 & 50 \\
\hline Preparatoria & 63 & 39 \\
\hline Instituto de investigación & 13 & 4 \\
\hline Oficinas & 62 & 23
\end{tabular}

HTA: humo de tabaco ambiental

presencia de HTA y HTA alto fue reportada por más de $25 \%$ y más de $10 \%$ de los trabajadores, respectivamente, observándose poca variabilidad en la prevalencia de exposición entre las restantes instituciones. No se observaron diferencias entre el reporte de fumadores y no fumadores (datos no presentados).

En el cuadro V se compara la clasificación de cumplimiento con los espacios libres de HTA según los criterios de monitoreo, inspección o reporte de los trabajadores. La inspección clasifica como libre de humo

\section{Cuadro V}

Clasificación de CUMPLIMIENTO CON ESPACIOS LIBRES DE HUMO DE TABACO UTILIZANDO MONITOREO, INSPECCIÓN O RePORTE de los trabajadores. MéXICO, 2007

\begin{tabular}{llll} 
Institución & Monitoreo & Inspección & Reporte de trabajadores \\
Hospital I & No & Cumple & No \\
\hline Hospital 2 & No & No & No \\
\hline Clínica de neumología & Cumple & Cumple & No \\
\hline Universidad I & Cumple & Cumple & No \\
\hline Universidad 2 & No & No & No \\
\hline Universidad 3 & No & No & No \\
\hline Universidad 4 & No & Cumple & No \\
\hline Preparatoria & Cumple & Cumple & No \\
\hline Instituto de investigación & Cumple & Cumple & Cumple \\
\hline Oficinas & No & No & No
\end{tabular}

de tabaco $60 \%$ de las instituciones, el monitoreo $40 \%$ y la encuesta 10 por ciento. La decisión habría sido la misma con monitoreo o inspección en $80 \%$ de los casos, con monitoreo o encuesta en $70 \%$ y con inspección o encuesta en 50 por ciento.

\section{Discusión}

En el presente estudio hemos comparado tres métodos independientes (monitoreo ambiental, inspección directa y reporte de los trabajadores) para clasificar espacios públicos de México como libres de humo de tabaco. Nuestros resultados indican que el monitoreo ambiental y la inspección directa son los métodos más útiles para la evaluación. En general, el monitoreo y la inspección mostraron buen acuerdo puesto que en $80 \%$ de los casos ambos métodos clasificaron el cumplimiento del mismo modo. Si consideramos el monitoreo ambiental como el método de referencia, la inspección habría clasificado erróneamente a 20\% de las instituciones. En los casos discrepantes los monitores detectaron concentraciones altas en lugares poco conspicuos, como sótanos o baños. El reporte de los trabajadores sobre la presencia de HTA y de niveles altos de HTA fue frecuente y poco discriminante, concordando débilmente con el monitoreo y pobremente con la inspección

En este estudio hemos desarrollado criterios preliminares para evaluar la exposición al HTA y discriminar cuándo un espacio puede ser considerado libre de HTA. Los criterios desarrollados, particularmente el punto de corte en $0.1 \mu \mathrm{g} / \mathrm{m}^{3}$ para el monitoreo, pretenden evitar casos donde los niveles de concentración detectados pudieran deberse a fuentes de contaminación externa. 
Esto de ninguna manera implica que consideremos que este nivel de exposición es seguro o recomendable. Consideramos que la meta para todos los espacios cerrados debe ser la nula detección de nicotina en $100 \%$ de las áreas monitoreadas. Sin embargo, también creemos que el proceso de evaluación debe contar con criterios capaces de discriminar espacios donde, a pesar de la posible contaminación por fuentes externas, se están tomando medidas efectivas para eliminar el consumo de tabaco en áreas interiores. Estudios a futuro deberán confirmar si las concentraciones de $0.1 \mu \mathrm{g} / \mathrm{m}^{3}$ y $0.5 \mu \mathrm{g} /$ $\mathrm{m}^{3}$ son puntos de corte suficientemente sensibles para identificar contaminación externa.

El monitoreo ambiental es uno de los métodos de evaluación más utilizados, prefiriéndose a otras opciones debido a su capacidad no sólo para detectar sino para cuantificar el HTA. ${ }^{3}$ La cuantificación permite la evaluación de la intensidad de la exposición, proporcionando un indicador de riesgo más sensible y posibilitando la comparación con indicadores de salud relevantes. ${ }^{19}$ De igual forma, la sensibilidad del monitoreo y su capacidad de vigilancia continua lo hacen un buen instrumento para evaluación. Sin embargo, su mayor costo es una limitación importante para utilizarlo de manera masiva. Dado que en los dos casos en que la inspección no detectó la presencia de tabaco las violaciones ocurrieron en lugares poco conspicuos, una posibilidad sería utilizar de manera selectiva el monitoreo ambiental continuo en áreas donde es más fácil encubrir el consumo de tabaco.

La utilidad de las inspecciones para evaluar el cumplimiento ha sido observada en comparaciones pre y postintroducción de leyes sobre espacios libres de humo de tabaco, ${ }^{10}$ aunque el número de estudios previos utilizando este método es escaso. Nuestro estudio muestra que la inspección directa es un método sensible y con buen acuerdo con el monitoreo ambiental. Sin embargo, en este estudio se realizaron cinco visitas a las áreas de interés cuando, de manera general, las visitas se efectúan al azar en un solo día. Si hubiésemos seguido esta estrategia, la probabilidad de observar al menos un fumador en las áreas de inspección en cualquiera de las visitas habría sido entre 20 y $60 \%$ de lo observado. La inspección detectaría con más frecuencia colillas y olor a humo de tabaco, aunque de acuerdo con nuestro criterio estas dos condiciones tendrían que observarse simultáneamente para ser consideradas indicadores de falta de cumplimiento.

Aunque algunos estudios han reportado un buen desempeño de los cuestionarios para predecir concentraciones de nicotina, ${ }^{20,21}$ las dificultades observadas en nuestro estudio para evaluar el cumplimiento usando cuestionarios han sido reportadas anteriormente. ${ }^{9,22} \mathrm{La}$ interpretación subjetiva que implica el autorreporte dificulta el uso de cuestionarios para obtener una evaluación objetiva y confiable, por lo que algunos estudios han propuesto indicadores menos susceptibles a la interpretación, como la presencia de señalización en áreas interiores.$^{23} \mathrm{La}$ falta de discriminación observada en los cuestionarios es desalentadora. Sin embargo, su evaluación se limitó a una sola pregunta que no define con claridad "área de trabajo", pudiendo interpretarse incluso como áreas exteriores. Esto que explicaría la alta prevalencia de reporte en instituciones donde ni la inspección ni el monitoreo detectaron HTA y la poca capacidad de discriminación del instrumento. Futuros estudios deberán evaluar la posibilidad de utilizar preguntas más precisas.

El presente estudio utiliza una muestra a conveniencia con potencial sesgo de selección que, de existir, generaría una muestra de lugares con menor frecuencia e intensidad de exposición, requiriendo mayor sensibilidad para la detección. Por tanto, los criterios desarrollados deberían tener el mismo desempeño en lugares con mayor frecuencia e intensidad de exposición.

La existencia de la LGCT, que establece la obligatoriedad de los espacios libres de humo de tabaco en los lugares de acceso público, no garantiza su cumplimiento. En la aplicación de la ley debe considerarse el desarrollo de un sistema de vigilancia epidemiológica de la exposición al HTA capaz de proveer información confiable y continua sobre la implementación de las disposiciones oficiales. Los criterios para evaluar el cumplimiento con la ley deben ser válidos, sensibles y acordes con las características nacionales y tener una fundamentación clara que explicite sus ventajas y limitaciones.

Basado en los resultados de este estudio la combinación del monitoreo ambiental con la inspección proporcionaría el método de clasificación más sensible. En este estudio se ha utilizado el monitoreo de $20 \%$ de las áreas interiores de espacios públicos y la inspección directa en cinco ocasiones durante una semana, lo que representa el mejor escenario de evaluación posible con los recursos disponibles para el estudio. Comparado con la inspección, la información derivada del monitoreo proporcionaría una dimensión más de evaluación, la cuantificación de la exposición, de mayor utilidad para evaluaciones de riesgo a la salud. Sin embargo, en estudios de vigilancia epidemiológica estos procedimientos difícilmente podrían realizarse con tanta extensión o frecuencia, por lo que la capacidad de detección de ambos métodos observada en este estudio podría ser menor. Asimismo, el costo de realizar cualquiera de estos procedimientos de manera exhaustiva y masiva debe considerarse cuidadosamente, requiriéndose un 
estudio de costo-efectividad para identificar la mejor estrategia.

\section{Recomendaciones}

Los reglamentos locales y federales para el control del tabaquismo deberían no sólo integrar las medidas de prevención y control conocidas, sino también definir los mecanismos para establecer un sistema de vigilancia epidemiológica de la exposición al HTA. Los resultados de nuestro estudio muestran que el uso simultáneo de inspecciones y monitoreo incrementaría la precisión en la detección de espacios libres de humo de tabaco y, potencialmente, coadyuvaría al cumplimiento de la reglamentación. En la vinculación académica con el servicio, sobresale el intento del INSP, y del LACOT, de transformar la experiencia en la cuantificación ambiental de nicotina en conocimiento científico vinculado a la utilidad comunitaria, mediante los siguientes elementos:

1. Un sistema nacional de vigilancia epidemiológica homogéneo que integre la cuantificación de nicotina ambiental permitiría caracterizar en forma permanente, objetiva y dinámica, la exposición a humo de tabaco en lugares públicos.

2. Puede brindar información para la aplicación de acciones correctivas de prevención, control y erradicación de exposición ambiental a humo del cigarro en espacios cerrados.

3. Puede ser vinculado con mayor precisión a información clínica de los trabajadores.

4. Tiene la capacidad potencial de establecer un gradiente de exposición.

5. Coadyuvaría a monitorear la dinámica de los cambios en la permisividad social posterior a la aplicación de la reglamentación respectiva.

6. Facilitaría la comparación en diversos momentos y contextos geográficos de México y la región.

7. Establecería mecanismos de decisión y control sanitario, considerando que su principal objetivo es la identificación temprana de la exposición.

8. Tiene el carácter de oportunidad, y es una evidencia de interacción entre la aplicación de la salud pública y su vinculación social.

9. Debe de contar con un mecanismo de difusión de información, tanto de los resultados obtenidos, como de sus acciones correctivas.

\section{Agradecimientos}

El presente estudio ha sido financiado a través del Fondo Sectorial de Investigación en Salud y Seguridad Social del Consejo Nacional de Ciencia y Tecnología de
México, con el proyecto "Exposición al Humo de Tabaco Ambiental en Lugares Públicos en las Principales Ciudades de México", clave SALUD-2005-01-14212, otorgado a Luz Myriam Reynales Shigematsu.

Tonatiuh Barrientos Gutiérrez ha contado con una beca Fogarty International Center Training Grant (3 D43 TW00644) durante el desarrollo de este artículo. El Dr. Gimeno es apoyado a través de una beca del National Institute on Aging (AG13196).

Este estudio se desarrolló en colaboración con los Centros de Integración Juvenil AC, con el apoyo del Dr. Víctor Manuel Guisa Cruz (director general), del Dr. Jaime Quintanilla Bendek (director nacional de Tratamiento y Rehabilitación), del Psic. Enrique Aceves Arce (coordinador regional B), de la C. Rocío Sánchez Villamar (coordinadora regional D), y del Dr. Otoniel Cárdenas Elizondo (coordinador regional F), quienes apoyaron en el proceso de convocatoria, reclutamiento y seguimiento local de las instituciones.

También agradecemos la participación de los señores Tito Alejandre Badillo e Ignacio Morales Pérez, quienes realizaron el trabajo de campo, así como de los químicos Ana Larissa Barbosa Sánchez, Abigail Flores Escartín, Lisset Orgaz Pérez y José Leopoldo Parada Cisneros, del LACOT del INSP, quienes efectuaron el análisis químico de los monitores de difusión pasiva.

\section{Referencias}

I. World Health Organization. WHO framework convention on tobacco control (WHO FCTC). Geneva: WHO, 2003.

2. Antuñano FJL. Firma y ratificación del Convenio Marco para el Control del Tabaco, de la Organización Mundial de la Salud. Salud Publica Mex 2004;46:273.

3. US Department of Health and Human Services. The health consequences of Involuntary Exposure to tobacco smoke: A report of the Surgeon General. Atlanta, GA: US Department of Health and Human Services, 2006.

4. Environmental Protection Agency. Respiratory Health Effects of Passive Smoking: Lung Cancer and Other Disorders. US Department of Health and Human Services, 1993.

5. World Health Organization. Policy recommendations on protection from exposure to second-hand tobacco smoke. Geneva: WHO, 2007. 6. Cámara de Senadores de los Estados Unidos Mexicanos. Ley General para el Control del Tabaco, México, 2008. Gaceta Oficial del Senado de la República 2008;198:115-123.

7. Alpert HR, Carpenter CM, Travers MJ, Connolly GN. Environmental and economic evaluation of the Massachusetts smoke-free workplace law. J Community Health 2007;32:269-28I.

8. Heloma A, Jaakkola MS. Four-year follow-up of smoke exposure, attitudes and smoking behaviour following enactment of Finland's national smoke-free work-place law. Addiction 2003;98: I III.

9. Borland R, Yong HH, Siahpush M, et al. Support for and reported compliance with smoke-free restaurants and bars by smokers in four countries: Findings from the International Tobacco Control (ITC) four country survey. Tob Control 2006;15(suppl 3):iii34-iii4l. 
10. Weber M, Bagwell D, Fielding J, Glantz S. Long term compliance with California's smoke-free workplace law among bars and restaurants in Los Angeles county. Br Med J 2003; 12:269.

II. Nagle AL, Schofield MJ, Redman S. Smoking on hospital grounds and the impact of outdoor smoke-free zones. Tob Control 1996;5:199-204.

12. Edwards R, Bullen C, O'Dea D, Thomson G, Waa A, Wilson N. After the smoke has cleared: Evaluation of the impact of a new smoke-free law. Tob Control 2008; 17:2.

13. Abrams SM, Mahoney MC, Hyland A, Cummings KM, Davis W, Song L. Early evidence on the effectiveness of clean indoor air legislation in New York State. Am J Public Health 2006;96:296-298.

14. Williams A, Peterson E, Knight S, Hiller M, Pelletier A. Survey of restaurants regarding smoking policies. J Public Health Manag Pract 2004; 10:35-40.

15. Leaderer BP, Hammond SK. Evaluation of vapor-phase nicotine and respirable suspended particle mass as markers for environmental tobacco smoke. Environ Sci Technol 1991;25:770-777.

16. Guerin MR, Jenkins RA, Tomkins BA. The Chemistry of Environmental Tobacco Smoke: Composition and Measurement. CRC Press, 2000.

17. Hammond SK, Leaderer BP. A diffusion monitor to measure exposure to passive smoking. Environ Sci Technol 1987;21:494-497.
18. Barrientos-Gutierrez T, Valdés-Salgado R, Reynales-Shigematsu LM, Navas-Acien A, Lazcano Ponce EC. Exposición involuntaria al humo de tabaco en lugares públicos de la ciudad de México. Salud Publica Mex 2007;43:S205-S2I2.

19. Goodman P, Agnew M, McCaffrey M, Paul G, Clancy L. Effects of the Irish smoking ban on respiratory health of bar workers and air quality in Dublin pubs. Am J Respir Crit Care Med 2007; 175:840-845.

20. Chan CC, Chen YC, Wang JD. Comparison of questionnaires, cigarette butt counts, and nicotine concentration measurements in predicting schoolchildren nicotine exposure. Bull Environ Contam Toxicol 1994:53:254-258.

2I. Coghlin J, Hammond SK, Gann PH. Development of epidemiologic tools for measuring environmental tobacco smoke exposure. Am J Epidemiol 1989;130:696-704.

22. Woodward A, Al-Delaimy W. Measures of exposure to environmental tobacco smoke. Validity, precision, and relevance. Ann N Y Acad Sci 1999;895:156- 172.

23. Gooding M, McAllister I. Evaluating compliance with Australia's first smoke-free public places legislation. Tob Control 1997;6:326-331. 